\title{
Comparing Emergency Department Gunshot Wound Data with Mass Casualty Shooting Reports
}

\author{
Andrew Walsh* \\ Health Monitoring, Pittsburgh, PA, USA
}

\section{Objective}

To determine whether mass casualty shooting events are captured via syndromic surveillance data.

\section{Introduction}

Shootings with multiple victims are a concern for public safety and public health. The precise impact of such events and the trends associated with them is dependent on which events are counted. Some reports only consider events with multiple deaths, typically four or more, while other reports also include events with multiple victims and at least one death. ${ }^{1}$ Underreporting is also a concern. Some commonly cited databases for these events are based on media reports of shootings which may or may not capture the complete set of events that meet whatever criteria are being considered.

Many gunshot wounds are treated in the emergency department setting. Emergency department registrations routinely collected for syndromic surveillance will capture all of those visits. Analysis of that data may be useful as a supplement to mass shooting databases by identifying unreported events. In addition, clusters of gunshot wound incidents which are not the result of a single shooting event but still represent significant public safety and public health concerns may also be identified.

\section{Methods}

Emergency department registration data was collected from hospitals via the EpiCenter syndromic surveillance system. Gunshotrelated visits were identified based on chief complaint contents using EpiCenter's regular expression-based classification system. The gunshot wound classifier attempts to exclude patients with preexisting wounds and shooting incidents involving weapon classes that are lesser concerns for public safety, such as nail guns and toy guns.

Gunshot-related visits were clustered by day of registration and separately by facility, by patient home zip code, and by patient home county. The largest clusters of each type were compared via manual search against media reports of shootings and against the Gun Violence Archive mass shooting database.

\section{Results}

A total of 23,132 gunshot-related visits were identified from 635 healthcare facilities from 2013 to 2015 . From these, the five largest clusters by facility, by zip code, and by county were identified. The clusters included 112 gunshot wounds in total, ranging in size from 4 to 12 with a median of 7 .

Of the 5 facility clusters, 5 had a corresponding media story and 2 were located in the shooting database. Of the 5 zip code clusters, 1 had a corresponding media story and none were located in the shooting database. Of the 5 county clusters, 4 had a corresponding media story and 1 was located in the shooting database.

\section{Conclusions}

Multiple gunshot wound patients being treated on the same day were not necessarily all shot during the same incident or by the same shooter. The information available in a syndromic surveillance feed does not allow for direct identification of the shooter or shooters. Given that limitation, a complete correspondence between clusters identified in syndromic surveillance data and mass shootings was not expected. The strong correlation between clusters and media coverage indicates that the news is a reasonable source for shooting data. The smaller overlap with the mass shooting database is likely due to the more stringent criteria required for an incident to qualify as a mass shooting.

It is still notable that the majority of gunshot clusters were not associated with any particular mass shooting incident. This serves as a reminder that mass shootings represent only a small portion of the total gun violence in the United States. Healthcare data represents a significant additional data source for understanding the complete impact of gun violence on public health and safety.

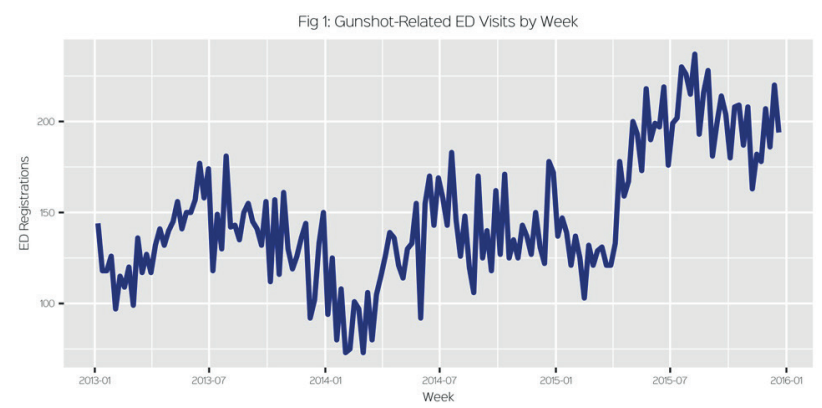

Weekly time series of gunshot-related emergency department visits

\section{Keywords}

mass shooting; gunshot wound; emergency department

\section{Acknowledgments}

We wish to thank our public health customers for funding support and data for this work.

\section{References}

1. Ingraham C. What makes a 'mass shooting' in America. Washington Post (Online). 2015 Dec 3:Wonkblog https://www.washingtonpost. com/news/wonk/wp/2015/12/03/what-makes-a-mass-shooting-inamerica/

\section{*Andrew Walsh}

E-mail: andy.walsh@hmsinc.com 